\title{
MaGrid: A Language-Neutral Early Mathematical Training and Learning Application
}

\author{
https://doi.org/10.3991/ijet.v13i08.8271 \\ Tahereh Pazouki $\left.{ }^{\varpi}\right)$, Véronique Cornu, Philipp Sonnleitner, \\ Christine Schiltz, Antoine Fischbach, Romain Martin \\ University of Luxembourg, Louxembourg \\ tahereh.pazouki@uni.lu
}

\begin{abstract}
Given that the use of educational technologies is increasingly popular in kindergarten and among young students, these technologies have been shown to be able to solve the difficulties (existing in school) that are either not possible to be solved in traditional schooling or cost a lot of time and resources. In this paper, we present a pedagogical training and learning application called MaGrid (Math on Grid), which has been developed to foster early mathematical skills in pre-schoolers. MaGrid is a tablet-based application, which provides a wide range of training tasks targeting fundamental mathematical concepts for the preschool level. The language-neutral property of MaGrid is an innovative aspect that makes MaGrid different from existing mathematical training applications. This property may reduce the barrier of language from mathematical education for second language learners encountered in multilingual school settings. MaGrid allows individual learning in an interactive way and provides real-time feedback. Moreover, MaGrid is capable of recording students' activities while working on the training tasks, which can help teachers and parents keep track of a student's progress in different tasks and observe potential training-related improvements over time. Finally, we describe a series of experiments carried out using the MaGrid application during special training and assessment sessions in several preschools in Luxembourg.
\end{abstract}

Keywords-Interactive systems. Individual learning. Training and learning application. Early mathematics. Language-neutral. Second language learners

\section{Introduction}

Although developing mathematical skills is key to succeed in educational systems [1], their importance is not limited to school. Quality of life as well as the financial situation have been shown to be related to mathematical achievements at school [2], [3]. Mathematical abilities at the beginning of elementary school are strongly predictive of later academic achievement [4]-[9]. In other words, children who failed to acquire sufficient knowledge of basic mathematics during the preschool years, such as counting and number knowledge, will not have a strong foundation to build up mathematics later on [4], [5], [10], [11]. This is due to the hierarchical nature of the devel- 
opment of mathematical skills. For instance, to solve the calculation " $3+2=5$ ", a student should have knowledge of: (a) the symbolic numerical system, (b) the sign "+" meaning adding and (c) the fact that adding two numbers leads to an exact number which is the sum of two operands [12].

Developing mathematical ability is a process between the teacher and learner, which requires an effective communication [13] and language, used to define mathematical concepts and ideas, playing a key role in this process. Moreover, the proficiency in the language of instruction exhibits a significant influence on learning mathematics [14], [15]. Accordingly, the learning of mathematics by second language learners (students whose primary language is different from the language of instruction) raises some critical issue. The learning of mathematics demands a variety of linguistic skills that second language learners may not have mastered $[16][16][16][16]$. Cummins [17] has hypothesized that 5 to 8 years may be required for the mastery of the linguistic skills necessary for academic pursuits in a second language.

Taken together, second language learners are at risk of obtaining a poor mathematical foundation and of lagging behind their native peers in formal schooling as they start with weaker math skills compared to their native peers (due to the language barrier) [14], [15]. This finding especially applies to countries with heterogeneous student populations and a high number of students with a migratory background, such as Luxembourg. In Luxembourg, $63.5 \%$ of surdents are non-native speakers of the Luxembourgish language (Ministère de l'Education nationale, de l'Enfance et de la Jeunesse, 2017). Consequently, given that Luxembourgish is the language of instruction in preschool classrooms, children as well as their teachers face major challenges.

To level non-native students with their native peers and thereby close potential performance gaps already present prior to formal schooling and working on numeracy deficits, an effective language-neutral early math intervention targeting the building blocks of early mathematical development is required.

"Language-neutral" material means that the instructional material does not contain any language-related input. Therefore, every child, independent of his or her proficiency in the actual language of instruction, should be able to benefit from languageneutral training tools.

The major building blocks of early mathematical development are described by Sarama and Clements [18], as: (a) visuo-spatial and geometric competencies and concepts and (b) numeric and quantitative concepts [18]. Research shows that young children are intuitively capable in these areas [19]-[21]. As a result, many school curricula, such as the Luxembourgish national school curriculum also emphasize the need to foster young children's abilities in these two broad domains [22].

In this paper, we present MaGrid, a training and learning application that was designed in a language-neutral way and especially targets the development of major building blocks of early mathematics. Hence, it aims at reducing potential language barriers in students with migration background and therefore at closing existing performance gaps in early mathematical education. In a second step, the results of two empirical intervention studies, that investigated the empirical effectiveness of the 
present application, are reported. We conclude by describing the limitations and the future work of MaGrid, as well as how it contributes to the field.

\subsection{Learning via tablet devices}

Challenges associated with multilingual school settings and heterogeneous student populations can successfully be faced using digital devices such as personal computers (PCs) and tablet devices. For example, digital devices allow presenting training material and instructions in visual format. This emphasis on visual, rather than verbal training material can thus reduce the language load and provide all children, regardless of their language background, with an opportunity to benefit from the training. Moreover, digital devices are able to provide an individual interactive training in which a student can repeat instructions as often as needed for their individual learning pace (as suggested by Pitchford)[23].

Among existing electronic devices, touch screen devices and more specifically tablet devices are becoming more and more popular in educational systems [24]-[27]. Tablet devices like iPads are mobile and lightweight while one could still have hundreds of applications on them. More importantly, touch screens are easier to work with for children with lower motor requirements than PCs (to use a PC a child needs to struggle with the keyboard and the mouse while keeping the eyes on the screen) [27], [28]. These reasons explain why pre-schoolers find tablet devices very motivational and easy to use [29], [30].

Therefore, tablet devices might qualify as the platform on which to run potential language-neutral training applications. They would optimally combine the possibility to visualize instructions and at the same time allow for an easy interaction with the program.

Although a multitude of so-called educational games to train mathematical skills exist - often only commercially available - they usually lack a scientific foundation and are meant to be played by the students on their own and not in school classrooms. Thus, there is a significant lack of applications making use of the advantages modern technology has to offer while at the same time being based on scientific research (i.e. training tasks in line with scientific findings and theories) and being optimized to be administered in classroom settings by teachers and educators[31].

\section{$2 \quad$ MaGrid $^{1}$}

MaGrid is a research-based early math training and assessment application designed to foster early mathematical skills. The application can be used either in classrooms in the school context, but it is also possible to use it independently from the school context at home. The main aim of implementing the MaGrid application was to foster young children's early mathematical abilities without relying on any lan-

\footnotetext{
${ }^{1}$ We should state that the MaGrid training application is not yet available for practical use, as the research on validating this tool is not completed, but interested researchers could contact us (tahereh.pazouki@uni.lu) to use this training application for research purposes.
} 
guage skills. MaGrid presents visuo-spatial and mathematical tasks and concepts visually, and allows children to have an interactive exploration to find the solution for the given task, and finally it verifies the user's solution and provides real-time feedback on the solution. MaGrid includes a large number of different tasks targeting different aspects of early mathematical abilities to maintain young children's interest and to provide them with a profound and flexible understanding of basic mathematical concepts. All training tasks have been developed in accordance with established developmental models of numerical cognition [32] and further findings from empirical research on visuo-spatial and numerical development.

\subsection{MaGrid features}

MaGrid has a number of distinguishing features: A) Language neutrality, B) Combination with external materials, C) User Handling and Task Management with QR Codes, D) Variety of tasks, and E) The built-in logging and monitoring system.

To the best of our knowledge, although there are a few academically motivated pedagogical training applications on early mathematical abilities such as 'Math Garden' [33] and 'Math Shelf' [34], none of these training applications offer all capabilities of MaGrid together.

Language neutrality. The main purpose of our work was to train early mathematical abilities using a language neutral approach. The exclusively visual design of the application is therefore independent of any language requirements such as text or voice-overs. This was made possible by visualizing the mathematical concepts and ideas, and by providing real-time feedback on the users' action while they are trying to solve a task and guiding them towards finding the solution. When a user starts working on a training task, the application plays a tutorial video, which shows a visual example of solving an instance of the selected task without any verbal explanation. When the video is over, the user is presented with the first level of the selected task category and can start interacting with the objects on the screen to explore the objective of the given task and find a solution for it. This process includes visual hints and sound effects, which assist the user without depending on any text. Similarly, as soon as the current task is solved, the user will be notified with visual effects and the next level will load on the screen.

Each type of training task comes with 30 to 60 levels of difficulty, starting from very simple ones to medium and more challenging ones. This way, students learn mathematical concepts intuitively, at their own pace and by doing examples.

The language neutral property makes early mathematical instruction intuitive and accessible to all children regardless of their language background or their proficiency in the language of instruction, and can thus be used in school settings with heterogeneous student populations. This has the further advantage of being able to be used in any classroom across the world, without requiring any modifications regarding language or instructions, which should meet all pre-schoolers' needs equally.

Combination with external material. MaGrid is not a solo application but it comes with external material, namely (paper) booklets. Booklets are supplementary physical books containing the templates of different tasks. The use of external materi- 
al does, on the one hand, augment the visual support provided during the learning activities, and on the other hand, it makes it more suitable for the classroom context. This approach allows teachers (or parents) to actively guide children's learning progress by providing them with the activities they consider the most appropriate.

User Handling and Task Management with QR Codes. To start using the application, each user needs to have a MaGrid Identity card (ID card) containing a unified Quick Response (QR) code. The ID cards will be used for user management and user identification. That means users can $\log$ in to the system by putting their ID card in front of the camera of the device and then they can start or resume solving tasks. Using QR codes for user management allows users to login to their account without typing any usernames or passwords: This is of critical importance for the usability of the training application, as our main users are pre-schoolers who are not yet able to read or write. Each training task has also an ID card with the corresponding QR code printed on it, so a user can start a task by scanning the ID card of the desired task.

QR codes are used to run the tasks as a means to structure the training and to give the teacher the possibility to decide which task pupils should work on. When a specific task $\mathrm{T}$ is to be worked on, the teacher distributes the ID card for task $\mathrm{T}$ to the pupils, which they then use for launching their tasks.

Variety of tasks. The present application includes 32 different number-specific (from non-symbolic tasks to symbolic tasks and mapping between different representations of numbers) and simple arithmetic task types. Beyond that, it provides 16 different visuo-spatial task types focusing on different aspects of visuo-spatial ability that have been linked to mathematical development (visuo-motor integration, visual perception, spatial visualization, shape recognition and differentiation). And, as mentioned earlier, each type of task comes with different difficulty levels. Overall, the number of task instances amounts to 2.5 thousand task instances which makes MaGrid a comprehensive training application.

Built-in logging and monitoring system. The use of the designed training application is not restricted to the sole purpose of training, but due to its built-in logging and monitoring system it can also be used as an assessment application. So apart from students who use the application for solving tasks, teachers and parents can also benefit from this application for evaluating user behavior in different visuo-spatial or mathematical tasks by monitoring their activity and their progress through the realtime monitoring mechanism.

While the user is working with the application, the system records all user actions into a $\log$ file. For each user action, the system generates a log message. Each log message contains the following information: The timestamp of an action, the ID of the logged-in user, task name, task level, type of task (selection, drawing, colouring, rotation, etc.), the ID of the selected shape (if the task type is selection) and the coordinates of touches. Logs are designed in a way that they are easy to read even before any preparation and they are simplifying post-processing and analysis of the recorded data. Easy to read logs and real-time monitoring will give a teacher the opportunity to identify the problems of students and to give them individual feedback and help.

By exploring log files, one can extract various levels of information. On a basic level, data can be summarized to give numeric values using descriptive statistics [35]. 
For example, a simple summary of a log file could be the number of solved items or the total time taken by a student(s) to solve a set of items, or another example could be comparing the average time of solving items among students. As explained before, each type of task includes several levels and these levels are different in terms of their difficulty (some are easier and some harder). Defining difficulties for the levels was based on our observation on previous evaluations of young students' performance in these tasks presented in paper and pencil. But the difficulty level of the tasks could also be based on the information recorded in log files from the intervention studies, with indicators like time spent in different levels and the number of errors made while solving the tasks.

On a more advanced level of mining the log file, the data can be used by researchers who seek to figure out if there exist any special patterns in user behaviour for solving the tasks [36], [37] and to eventually understand how students learn, using data mining techniques.

\subsection{How does MaGrid work?}

To illustrate how MaGrid works, a series of screenshots taken from the initial screen until the completion of a task will be shown and explained. Figure 1 shows the main screen of the application, which is the initial starting point. Through this screen, users are able to log into the system by touching on the avatar picture on the screen and then scanning the QR code on their ID cards. When they are logged in, they can start playing/solving the tasks by touching on the "Play" button and then scanning the QR code printed on the task's ID card. After selecting a task, the screen transitions to the tutorial video screen (where an instruction video on the selected task type will be played) shown in Figure 2. When the tutorial video is finished, the screen transitions to the task screen where the user can start working on the training tasks.

\subsection{The Underlying Grid}

The name of the presented training application, MaGrid, already contains the central functional element of the system in which all mathematical tasks are implemented: the grid. A grid is a set of nodes (10 columns, base-ten system) that gives the user the possibility to draw/ delete lines on it. Figure 3 shows a screenshot of an empty grid. Users can draw a line on the grid by connecting any two points on the grid (grid nodes), delete a drawn line from the grid, move a drawn shape over the grid and delete a selected shape from the grid. The grid is used to visualize the base-ten system and to better structure the space. Furthermore, the use of the grid for drawing activities reduces the motor-demand of drawing (as they only need to connect the dots on the grid). As fine-motor skills are still developing in young students, this approach should help them to complete the visuo-spatial tasks. The grid should facilitate the drawing process - even of more complex shapes - for young children. In some of the tasks, the grid nodes are partially or completely invisible, so they may not be seen in all of the application's screenshots. 
Based on user actions, all visuo-spatial and mathematical tasks in MaGrid can be categorized into three separate groups: selection, drawing, and movement. In the 'selection' category of tasks, a set of shapes will appear on the screen and the user has to select a shape/shapes in order to complete the task. For tasks of the 'drawing' type, the user has to draw a set of lines on the grid in order to complete or reproduce a shape or to complete a pattern. And in 'movement'-based tasks, a set of shapes will appear on the screen and the user should move the shapes on screen (with drag and drop) in order to place them into a certain order to complete the task.

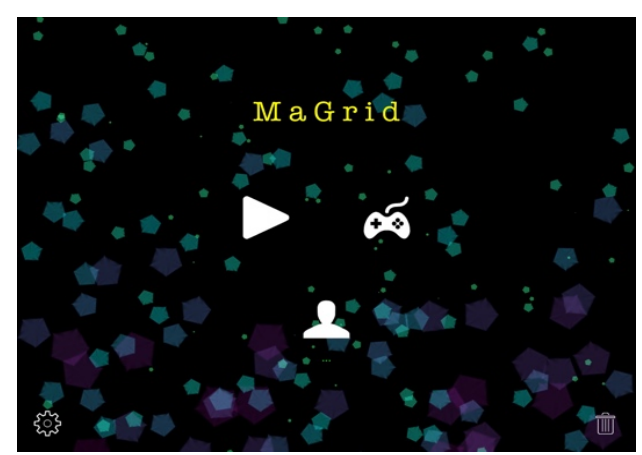

Fig. 1. Main screen

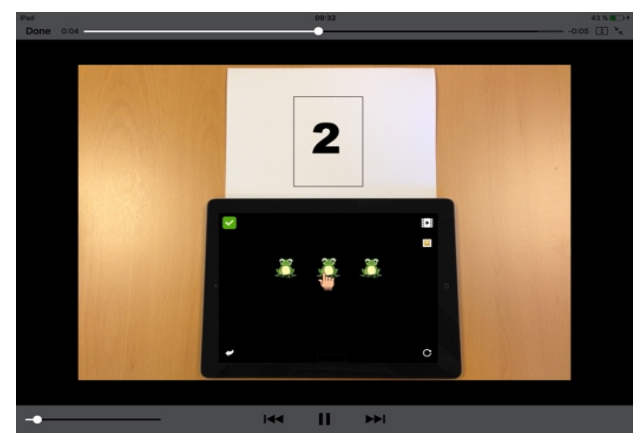

Fig. 2. Video screen

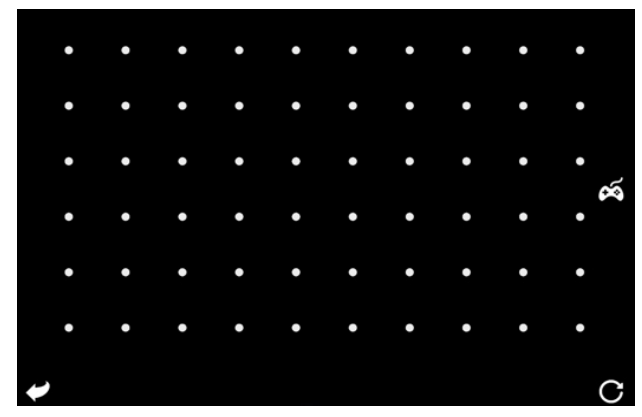

Fig. 3. An empty grid 


\subsection{Theoretically Derived Tasks}

A large number of different tasks targeting different aspects of early VSA (that have been linked to math development) and mathematical abilities were designed and developed in the MaGrid application. The tasks that MaGrid contains can be categorized into two main categories: visuo-spatial and numerical tasks.

Visuo-Spatial Tasks. As we mentioned before, a variety of visuo-spatial training tasks, tapping into different aspects of visual and spatial abilities, were designed and developed in the MaGrid application. Large percentages of our tasks in this category are focused on different tasks of visual perception. Besides visual perception tasks, our training application contains a number of visuo-motor integration (VMI) tasks (characterized by the task's motor component). VMI tasks not only require the visual processing of information but also a motor output and the coordination between the two. Their role for math achievement has been highlighted in different studies [38], [39].

Whereas tasks of visual perception have a comparably low motor component (e.g. children only have to select a target figure), VMI tasks require the child to draw parts of a shape (e.g. figure completion and shape closing) or entire shapes (e.g. copying tasks). All the tasks integrated in this part of the training are summarized under the umbrella term of "Visuo-Spatial Ability".

The training tasks are based on the commonly used tasks for training and assessing visuo-perceptive abilities in children [40], [41]. These abilities have been shown to be predictive of children's mathematical abilities in first grade [42]. Therefore, we aimed to adapt these tasks and to develop training tasks tapping into the same construct. The respective constructs, as defined by Büttner and colleagues [40] or Frostig and colleagues [41] are: spatial relations, form consistency, and position in space. Furthermore, we aimed to align our training application with the requirements of the national school curriculum. Therefore, the training application includes different visuo-spatial tasks that are specifically targeting competencies and related activities as described in the national school curriculum, such as symmetry, copying geometric shapes, completing patterns [22]. Table 1 provides: (a) an example of each category of visuo-spatial tasks with a brief description of them, (b) the number of task types in MaGrid from this category and (c) the motor component of the tasks in the category.

Table 1. Visuo-Spatial Tasks with a brief description

\begin{tabular}{|c|l|c|c|}
\hline Task Type & \multicolumn{1}{|c|}{ Name and description of an example task } & $\begin{array}{c}\text { Motor Com- } \\
\text { ponent }\end{array}$ & $\begin{array}{c}\text { Number of task types } \\
\text { from this category }\end{array}$ \\
\hline $\begin{array}{c}\text { Position in } \\
\text { Space }\end{array}$ & $\begin{array}{l}\text { Mental Rotation: responder has to select a shape } \\
\text { from the screen which is the rotated version of the } \\
\text { shape depicted in the booklet. (Figure 4) }\end{array}$ & Low & 5 \\
\hline $\begin{array}{c}\text { Form Con- } \\
\text { sistency }\end{array}$ & $\begin{array}{l}\text { Put together: an incomplete shape is shown on the } \\
\text { left side of the screen, and a few smaller shapes on } \\
\text { the right side of the screen and the responder should } \\
\text { select a shape from the right side which completes } \\
\text { the shape on the left. (Figure 5) }\end{array}$ & Medium & 4 \\
\hline $\begin{array}{c}\text { Spatial Rela- } \\
\text { tions }\end{array}$ & $\begin{array}{l}\text { Symmetry: responder has to draw a symmetric copy } \\
\text { of the shape on the screen. (Figure 6) }\end{array}$ & High & 7 \\
\hline
\end{tabular}




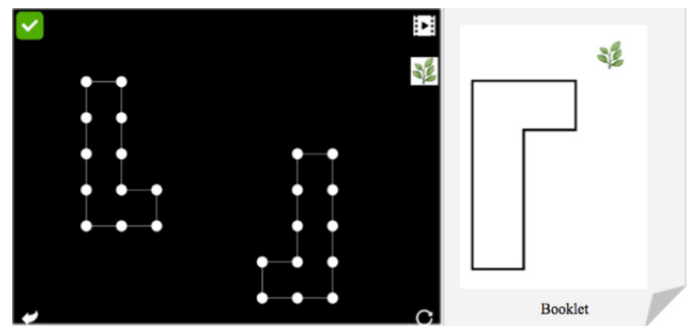

Fig. 4. Mental rotation

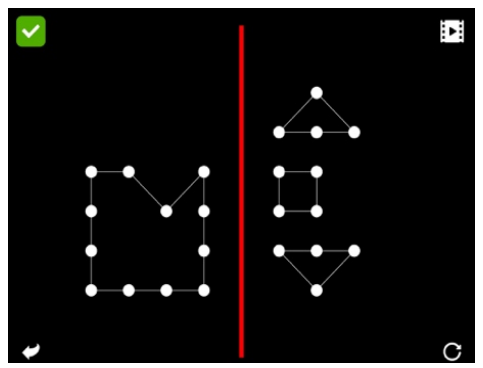

Fig. 5. Put together

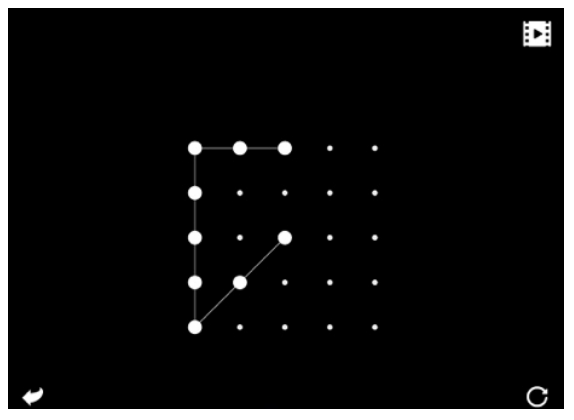

Fig. 6. Symmetry

Numerical Tasks. The number-specific tasks target different aspects of basic math competencies. The theoretical framework for designing and developing these tasks has been set by the four-step developmental model of numerical cognition [12]. In this developmental model, four different forms of number knowledge are distinguished: quantitative knowledge, verbal number knowledge, Arabic number knowledge and sequential knowledge.

The numerical tasks in MaGrid focus on these four components and the mapping between different representations of numbers (mapping between the first three steps in a developmental model). Table 2 provides: (a) a small subset of numerical training tasks with a short description of them, these tasks are categorized based on their symbolic or non-symbolic aspect and (b) the number of task types in MaGrid from this category. 
Table 2. Numerical tasks and their description

\begin{tabular}{|c|l|c|}
\hline Task Type & \multicolumn{1}{|c|}{ Name and description of an example task } & $\begin{array}{c}\text { Number of task types } \\
\text { from this category }\end{array}$ \\
\hline Non-Symbolic & $\begin{array}{l}\text { Pick fruits from 2 trees: responder has to pick the quantity of } \\
\text { fruits from each tree as depicted in the booklet. (Figure 7) }\end{array}$ & 16 \\
\hline Symbolic & $\begin{array}{l}\text { Frog Race: responder has to put the frogs (holding a num- } \\
\text { ber) on the correct position on the screen. (Figure 8) }\end{array}$ & 7 \\
\hline Mapping & $\begin{array}{l}\text { Aquarium: responder has to put the number of fishes in the } \\
\text { aquarium based on the number written in the booklet. (Fig- } \\
\text { ure 9) }\end{array}$ & 9 \\
\hline
\end{tabular}
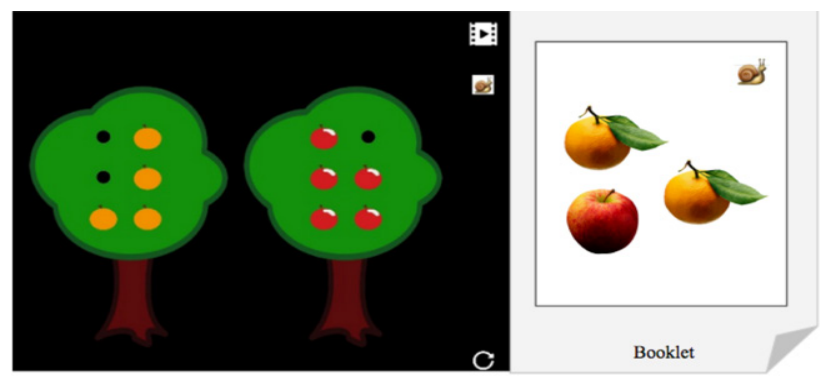

Fig. 7. Pick fruits from two trees

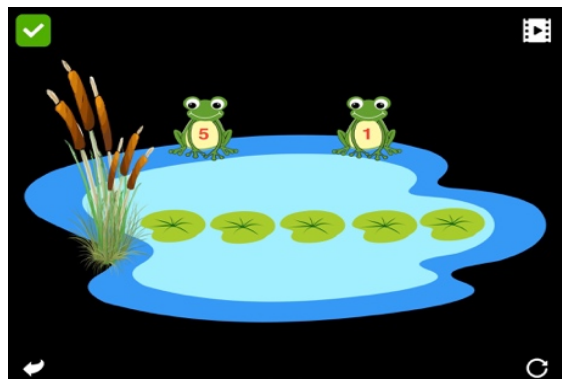

Fig. 8. Frog race

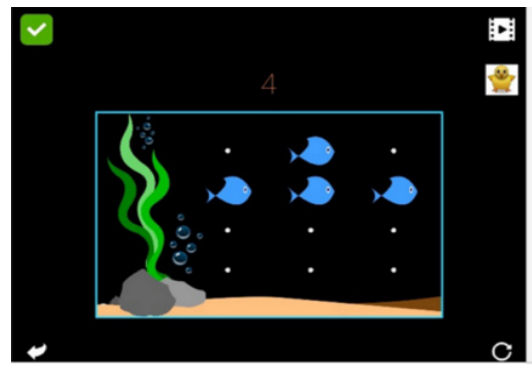

Fig. 9. Aquarium 
Free Play mode. Note that MaGrid also contains an additional mode called "Free Play mode" that is apart from the visuo-spatial and number-specific tasks. In this mode, the user has an empty grid to draw and delete lines and shapes without any limitations to explore the basics of the application. In addition to the event logging mechanism, which is always activated in this application for recording user activities, the application takes screenshots of users' drawings in this mode as shown in Figure 10. This mode can either be used to make the students familiar with the functioning of the grid or to study students' behavior in an unguided situation.
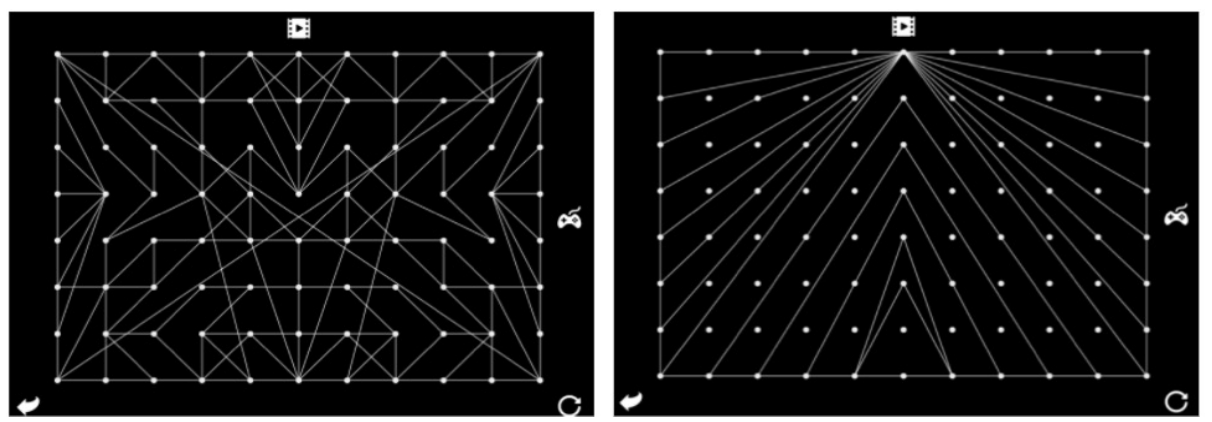

Fig. 10.Screen shots of user drawing in free play mode

\section{Empirical studies on MaGrid's effectiveness}

Until now, the MaGrid application has been used in two main research studies to survey its scientific effectiveness. In the first study, the MaGrid application was deployed in a study aiming to assess the effects of the visuo-spatial training tasks on pre-schoolers [43]. In this study, children from five classrooms worked with the MaGrid application twice a week over a period of ten weeks. We are presenting our effect sizes using Hedges'g, as our sample sizes in the intervention group and the control group were not identical. Results revealed that children who used the application over this period made significant gains in VSA with effect sizes (Hedges'g) ranging between 0.62 and 0.74 (these effect sizes can be considered as medium) [16], compared to children from classrooms who did not use the application. These results were the first empirical evidence, not only for the effectiveness of the training application, but also for the feasibility of implementing this application in an authentic school setting. The interested reader may refer to the published research paper for a detailed description of the study design and results [43].

In the second empirical intervention study, the MaGrid application was at the core of a comprehensive language-neutral mathematics training. This study assessed the training's effects on young second-language learners' early mathematical abilities [44]. As has been discussed previously, second-language learners are at risk of lagging behind their native peers in basic mathematical abilities, already during the preschool [14], [15]. For this purpose, a randomized-controlled trial design was implemented over the two years of preschool to investigate the effects of this training. 186 
children took part in this study, of which half participated in the language-neutral mathematics training, whereas the other half participated in a language intervention and was considered the control group. After these two school years, children who participated in the early mathematics training performed significantly better on several different measures of early mathematical abilities. Results revealed significant intervention effects on different measures of VSA with effect sizes (Hedges'g) ranging between 0.37 and 0.47 and on number specific skills with effect sizes ranging between 0.30 and 0.46 (these effect sizes can be considered small to medium) [16].

Taken together, these first empirical results are promising and show that the MaGrid application can be effective for all pre-schoolers, including traditionally underserved populations (second-language learners) and that it can be used on a classroom basis, but further research is still necessary to validate this training application in a more comprehensive way.

\section{Conclusion and outlook}

The importance of early numerical abilities to later mathematics achievement is clear[1], [7], [9] . MaGrid uses current technologies to visualize early numerical concepts and provides real-time feedback on students' actions to assist learning intuitively. The above-mentioned experiments provide first evidence that the MaGrid application would be an effective method for fostering early mathematical and numerical skills in pre-schoolers. They further show that this innovative approach, which is characterized on one hand by the language-neutral property (using visual material) and on the other hand by the proper use of tablet devices in school (using QR codes), is effective and can be integrated into everyday school life. This approach should be especially promising for schools with a heterogeneous student population with diverse language backgrounds to provide equal access to effective math education to all students.

A limitation of MaGrid in the current version of the application is that the training tasks are non-adaptive. In the current version, tasks are not yet adapted to the knowledge level of the student in that specific type of task. However, it is envisaged to include an adaptive feature in the future versions. The second plan for future work involves the integration of a data analysis tool into the MaGrid application. An integrated data analysis unit would perform processing operations in real-time and provide summarized result sets for the teacher in the form of tables and graphs at various stages.

We would like to remark that research on the effectiveness of this language-neutral early mathematics training and learning application has only just begun. Many questions on the effectiveness of the training program remain to be answered and factors related to the observed training effects need to be disentangled. 


\section{$5 \quad$ References}

[1] N. C. Jordan, J. Glutting, and C. Ramineni, "The importance of number sense to mathematics achievement in first and third grades," Learn. Individ. Differ., vol. 20, no. 2 , pp. 82-88, 2010. https://doi.org/10.1016/j.lindif.2009.07.004

[2] G. J. Duncan et al., "School readiness and later achievement.," Dev. Psychol., vol. 43, no. 6, pp. 1428-1446, 2007. https://doi.org/10.1037/0012-1649.43.6.1428

[3] S. J. Ritchie and T. C. Bates, "Enduring links from childhood mathematics and reading achievement to adult socioeconomic status.," Psychol. Sci., vol. 24, no. 7, pp. 1301-8, 2013. https://doi.org/10.1177/0956797612466268

[4] K. Bodovski and G. Farkas, "Mathematics growth in early elementary school: The roles of beginning knowledge, student engagement, and instruction," Elem. Sch. J., 2007. https://doi.org/10.1086/525550

[5] B. M. Casey, E. Dearing, A. Dulaney, M. Heyman, and R. Springer, "Young girls' spatial and arithmetic performance: The mediating role of maternal supportive interactions during joint spatial problem solving," Early Child. Res. Q., 2014. https://doi.org/10.1016/ j.ecresq.2014.07.005

[6] C. Hornung, C. Schiltz, M. Brunner, and R. Martin, "Predicting first-grade mathematics achievement: The contributions of domain-general cognitive abilities, nonverbal number sense, and early number competence," Front. Psychol., vol. 5, no. APR, pp. 1-18, 2014. https://doi.org/10.3389/fpsyg.2014.00272

[7] N. Jordan, D. Kaplan, and C. Ramineni, "Early math matters: kindergarten number competence and later mathematics outcomes.," Developmental, 2009.

[8] M. Papic, "Promoting repeating patterns with young children - More than just alternating colours!," Apm, 2007.

[9] T. W. Watts, G. J. Duncan, R. S. Siegler, and P. E. Davis-Kean, "What's past is prologue: Relations between early mathematics knowledge and high school achievement," Educ. Res., vol. 43, no. 7, pp. 352-360, 2014. https://doi.org/10.3102/0013189X14553660

[10] E. A. Gunderson, G. Ramirez, S. L. Beilock, and S. C. Levine, "The relation between spatial skill and early number knowledge: The role of the linear number line.," Dev. Psychol., 2012. https://doi.org/10.1037/a0027433

[11] J. A. Lefevre et al., "Pathways to Mathematics: Longitudinal Predictors of Performance," Child Dev., 2010. https://doi.org/10.1111/j.1467-8624.2010.01508.x

[12] M. Von Aster and R. S. Shalev, "Number development and developmental dyscalculia," Dev. Med. Child Neurol., 2007. https://doi.org/10.1111/j.1469-8749.2007.00868.x

[13] R. W. Morris, "Interactions between linguistics and mathematical education," in Report of a symposium by UNESCO-CEDO-ICMI. Nairobi, 1974.

[14] T. Kleemans, E. Segers, and L. Verhoeven, "Cognitive and linguistic precursors to numeracy in kindergarten: Evidence from first and second language learners," Learn. Individ. Differ., vol. 21, no. 5, pp. 555-561, 2011. https://doi.org/10.1016/j.lindif.2011 .07 .008

[15] P. Bonifacci, V. Tobia, L. Bernabini, and G. M. Marzocchi, "Early literacy and numeracy skills in bilingual minority children: Toward a relative independence of linguistic and numerical processing," Front. Psychol., vol. 7, no. JUL, pp. 1-14, 2016. https://doi.org/10.3389/fpsyg.2016.01020

[16] G. J. Cuevas, "Mathematics Learning in English As a Second Language," J. Res. Math. Educ., 1984. https://doi.org/10.2307/748889

[17] J. Cummins, "The entry and exit fallacy in bilingual education," NABE J., vol. 4, no. 3, pp. 25-59, 1980. https://doi.org/10.1080/08855072.1980.10668382 
[18] J. Sarama and D. H. Clements, "Building Blocks for early childhood mathematics," Early Child. Res. Q., 2004. https://doi.org/10.1016/j.ecresq.2004.01.014

[19] J. D. Bransford, A. L. Brown, R. R. Cocking, W. National Academy of Sciences - National Research Council DC.Commission on Behavioral and Social Sciences and Education., M. S. Donovan, and J. W. Pellegrino, How People Learn: Brain, Mind, Experience and School. 2000. 2000.

[20] D. H. Clements, "Geometric and spatial thinking in young children," Mathematics in the early years. 1999.

[21] H. P. Ginsburg, A. Klein, and P. Starkey, The Development of Children's Mathematical Thinking: Connecting Research with Practice. 1998.

[22] Ministère de l'Education nationale et de la Formation professionnelle, "Plan d'études école fondamentale," Luxembourg, Luxembourg, 2011.

[23] N. J. Pitchford, "Development of early mathematical skills with a tablet intervention: a randomized control trial in Malawi," Front. Psychol., 2015. https://doi.org/10.3389/ fpsyg.2015.00485

[24] K. Goodwin, "Use of Tablet Technology in the Classroom," Educ. Communities, 2012.

[25] O. T. Murray and N. R. Olcese, "Teaching and Learning with iPads, Ready or Not?," TechTrends, 2011. https://doi.org/10.1007/s11528-011-0540-6

[26] V. Rideout, "Zero to eight: Children's media use in America 2013," 2013.

[27] J. L. Tahnk, "Digital milestones: Raising a tech-savvy kid," Parent. Early Years, vol. 25, pp. 78-84, 2011.

[28] L. Z. Cooper, "Developmentally Appropriate Digital Environments for Young Children," Libr. Trends, 2005. https://doi.org/10.1353/lib.2006.0014

[29] R. Flewitt, D. Messer, and N. Kucirkova, "New directions for early literacy in a digital age: The iPad," J. Early Child. Lit., 2014.

[30] M. R. Boddum, "Plugged in: A focused look at parents' use of smartphones among children 2-5 years of age," Mills College, 2013.

[31] K. Hirsh-Pasek, J. M. Zosh, R. M. Golinkoff, J. H. Gray, M. B. Robb, and J. Kaufman, "Putting Education in 'Educational' Apps," Psychol. Sci. Public Interes., 2015.

[32] M. G. von Aster and R. S. Shalev, "Number sense and developmental dyscalculia," Dev. Med. Child Neurol., 2007. https://doi.org/10.1111/j.1469-8749.2007.00868.x

[33] M. and others Straatemeier, "Math Garden: A new educational and scientific instrument," University of Amsterdam, Amsterdam, 2014.

[34] J. Schacter et al., "Math Shelf: A randomized trial of a prekindergarten tablet number sense curriculum.," Early Educ. Dev., 2016. https://doi.org/10.1080/10409289.2015. 1057462

[35] C. Romero, S. Ventura, V. S. Rita, and M. Pechenizkiy, Handbook of Educational Data Mining. 2011.

[36] J. Mostow and J. Beck, "Some useful tactics to modify, map and mine data from intelligent tutors," Nat. Lang. Eng., 2006. https://doi.org/10.1017/S1351324906004153

[37] C. Romero and S. Ventura, "Educational data mining: A survey from 1995 to 2005," vol. 33, pp. 135-146, 2007.

[38] J. M. Sortor and M. T. Kulp, "Are the results of the beery-buktenica developmental test of visual-motor integration and its subtests related to achievement test scores?," Optom. Vis. Sci., 2003. https://doi.org/10.1097/00006324-200311000-00013

[39] S. Pieters, A. Desoete, H. Roeyers, R. Vanderswalmen, and H. Van Waelvelde, "Behind mathematical learning disabilities: What about visual perception and motor skills?," Learn. Individ. Differ., 2012. https://doi.org/10.1016/j.lindif.2012.03.014 
[40] G. Büttner, W. Dacheneder, W. Schneider, and K. Weyer, Frostigs Entwicklungstest der visuellen Wahrnehmung - 2, Hogrefe. Göttingen, 2008.

[41] M. Frostig, D. W. Lefever, and J. R. B. Whittlesey, "a Developmental Test of Visual Perception for Evaluating Normal and Neurologicallyhandicapped Children," Percept. Mot. Skills, vol. 12, no. 3, pp. 383-394, 1961. https://doi.org/10.2466/pms.1961.12.3.383

[42] M. Wantz, R. Martin, and C. Schiltz, "Numerical competencies of young children,” 2007.

[43] V. Cornu, C. Schiltz, T. Pazouki, and R. Martin, "Training early visuo-spatial abilities: A controlled classroom-based intervention study," Appl. Dev. Sci., 2017. https://doi.org/10.1080/10888691.2016.1276835

[44] V. Cornu, T. Pazouki, C. Schiltz, S. Ugen, C. Engel, R. Martin, Breaking the language barrier - A comprehensive and language-neutral early mathematics training for languageminority pre-schoolers" (in preparation).

\section{$6 \quad$ Authors}

Tahereh Pazouki is a PhD student at the University of Luxembourg at the Luxembourg Centre for Educational Testing (LUCET) working on the development of the MaGrid application. She received her Master degree in computer science from the University of Luxembourg with a specialization in data mining and knowledge discovery.

Véronique Cornu is a PhD student at the University of Luxembourg at LUCET working on the development of the MaGrid application. She received her Master degree in psychology from Saarland University (Germany) with a specialization in neuropsychology and clinical psychology.

Philipp Sonnleitner, affiliated to The Luxembourg Centre for Educational Testing (LUCET), University of Luxembourg, Dr in educational psychology from the Freie University of Berlin (Germany) in 2015. His academic research focuses on psychological and educational assessment. He is currently applying his expertise to the Luxembourg school monitoring program.

Christine Schiltz is Professor of cognitive neuroscience at the University of Luxembourg. She specializes in cognitive development and number processing. She is currently the head of cognitive science and assessment (COSA) institute and of the cognitive neuroscience (CNS) research group at the University of Luxembourg.

Antoine Fischbach, Dr in educational psychology from the University of Trier. He is specialized in large-scale educational assessment and he is an expert in the Luxembourg school system. He is currently the acting director of LUCET.

Romain Martin is Professor of psychology and empirical educational research at the University of Luxembourg. He specializes in cognitive abilities, educational measurement and computer-assisted testing. He is currently the vice-president for academic affairs at the University of Luxembourg.

Article submitted 19 January 2018. Resubmitted 30 March 2018. Final acceptance 11 July 2018. Final version published as submitted by the authors. 